\title{
Christliche Religionen und Masssenmedien in Brasilien
}

won Ari Pedro Oro

Der vorliegende Artikel ${ }^{1}$ will einen informativen Überblick über das Verhältnis der verschiedenen christlichen Kirchen und Denominationen im Brasilien der Gegenwart zum System der modernen Massenmedien vermitteln. Dieses Thema wird in sechs Punkten angegangen. In den ersten beiden Abschnitten werden ein kurzes Panorama der Religionen in Brasilien (1.) und einige Grunddaten und -fakten zur Geschichte und Gegenwart der brasilianischen Medienlandschaft (2.) entfaltet, bevor dann näher auf den Umgang und den Gebrauch der Massenmedien durch die katholische Kirche (3.), durch die traditionellen protestantischen Kirchen (4.) und durch die im Verlauf des 20. Jahrhunderts entstandenen Pfingstkirchen und das sog. Neupfingstlertum (5.) eingegangen wird. In einem abschließenden Fazit (6.) werden die wichtigsten Ergebnisse dieses Überblicks in einigen knappen Aussagen zusammengefaßt, um die Gemeinsamkeiten und Unterschiede im religiöskirchlichen Umgang mit dem breiten Spektrum der modernen Massenmedien auf den Punkt zu bringen.

Angesichts der geradezu kontinentalen Größe Brasiliens und der kaum noch überschaubaren Menge an statistischem Datenmaterial kann dabei freilich kein umfassendes Gesamtbild, sondern nur ein erster anschaulicher Einblick in die aktuelle Präsenz der christlichen Religionen auf dem massenmedialen Markt des größten lateinamerikanischen Landes gegeben werden.

\section{Ein Panorama der Religionen in Brasilien}

Der von den portugiesischen Eroberern eingeführte Katholizismus, der zwischen 1824 und 1889 auch als offizielle Staatsreligion fungierte, bildete unangefochten die hegemoniale Religion Brasiliens, bevor er sich in den letzten Jahrzehnten zunehmend mit der wachsenden Konkurrenz protestantischer, vor allem pfingstlerisch ausgerichteter Kirchen und Bewegungen auseinanderzusetzen hat. Dennoch weisen die Volkszäh-

PhD Ari Pedro Oro ist Professor für Sozialanthropologie an der Bundesuniversität von Rio Grande do Sul in Porto Alegre (Brasilien).

1 Redaktionell gekürzte und vom Autor aktualisierte Fassung eines erstmals in Social Compass (Louvain) 39 (1992) 3, 423-434 in französischer Sprache erschienenen Beitrages (Übers.: H.-J. Große-Kracht). 
lungen bis heute einen sehr hohen Anteil an Brasilianern aus, die sich als katholisch bezeichnen: 1990 waren es noch $85 \%$.

Der brasilianische Episkopat ist nach Italien und den USA der drittgrößte der Welt. In ihrer politischen Orientierung teilen sich die 370 Bischöfe Brasiliens grob in drei Richtungen auf, wobei der von etwa 60 bis 70 Bischöfen gebildeten konservativen Richtung eine ähnlich starke progressive Richtung gegenübergestellt werden kann, während die große Mehrheit der brasilianischen Bischofskonferenz allgemein als moderat eingestuft wird.

Im Hinblick auf die religiöse Glaubenspraxis ist in der brasilianischen Kirche zwischen einem offiziellen und einem populären Katholizismus zu unterscheiden, denn unabhängig vom ersteren, vor allem am Kerus und an der kirchlichen Sakramentenpraxis ausgerichteten Katholizismus hat sich seit langem eine vom einfachen Volk ausgeübte religiöse Praxis durchgesetzt, in deren Zentrum verschiedene kultische Formen der Hejligenverehrung stehen. Zum populären Katholizismus, der in einem oppositionellen Ergänzungsverhältnis zur offiziellen kirchlichen Religion steht, rechnet man ca. $70 \%$ der brasilianischen Katholiken.

Das Feld des Protestantismus ist in Lateinamerika komplex und unübersichtlich, so daß klare und zuverlässige Typologien nur schwer aufzustellen sind. Üblich ist jedoch die Unterscheidung zwischen dem historischen und dem pfingstlerischen (pentekostalistischen) Protestantismus geworden. Der historische Protestantismus fungiert als Sammelbegriff für die unmittelbar auf die Reformation zurückgehenden Kirchen der Lutheraner, Methodisten, Baptisten, Presbyterianer, Episkopalisten und Kongregationalisten, während sich der Begriff des pentekostalistischen Protestantismus für die sog. "Sekten"3 eingebürgert hat, die aus den nordamerikanischen religiösen Erweckungsbewegungen des frühen 20. Jahrhunderts hervorgegangen sind. $\mathrm{Zu}$ den wichtigsten pfingstlerischen Sekten in Brasilien gehören die ,Assembléia de Deus', die ,Congregação Cristã do Brasil' und die ,Igreja do Evangelho Quadrangular'. Einen besonderen Entwicklungsschub erleben die Pfingstbewegungen seit Beginn der 50er Jahre mit dem Aufkommen des sog. autonomen Pentekostalismus bzw. des Neopentekostalismus.

\section{Der Protestantismus in seinen verschiedenen Ausprägungen}

2 Der Anteil der Katholiken an der brasilianischen Gesamtbevölkerung ist kontinuierlich rückläufig. Ihre Zahl verminderte sich von $99,7 \% \mathrm{im}$ Jahr 1872 über 98,9\% im Jahr 1890, 95,0\% im Jahr 1940, 93,5\% im Jahr 1950 auf 91,8\% im Jahr 1970, 88,4\% im Jahr 1980 und 85,0\% im Jahr 1990. Die 1952 gegründete brasilianische Bischofskonferenz (CNBB) geht davon aus, daß in letzter Zeit jährlich etwa 600.000 Gläubige die katholische Kirche verlassen.

3 Dieser Begriff soll hier nicht in seinem geläufigen pejorativen, sondern nur in einem analytischen Sinn verstanden werden. 
erreicht insgesamt ca. 17\% der brasilianischen Bevölkerung. Dies entspricht in absoluten Zahlen in etwa einer Anhängerschaft von 30 Mio. Brasilianern, wobei die Pfingstbewegung ca. $60 \%$ des Gesamtprotestantismus ausmacht.

Ein drittes wichtiges Element im Bereich der brasilianischen Religionen bilden die sog. religiões mediúnicas, die sich zum einem aus den afrobrasilianischen Religionen und zum anderen aus dem auf die Lehre von ,Allan Kardec' (Hippolyte Denizard Rivail, 1804-1869) zurückgehenden kardecistischen Spiritismus zusammensetzen. $\mathrm{Zu}$ den ersteren gehören etwa der Candomblé und die Umbanda, die afrikanischen Ursprungs sind und ihre Anhängerschaft heute in verschiedenen ethnischen und sozialen Klassen und Gruppen finden. Der kardecistische Spiritismus wurde dagegen in der zweiten Hälfte des 19. Jahrhunderts aus Frankreich eingeführt und findet seine Anhänger lediglich in den sozialen Mittelschichten. Die afrobrasilianischen Kulte zählen heute etwa 50 Mio. Teilnehmer, während die Lehren von ,Allan Kardec' heute etwa 7 Mio. Anhänger finden.

Neben diesen größeren Religionen gibt es in Brasilien in kleinerem Ausmaß auch fernöstliche Religionen wie "Hare Krishna", 'Igreja da Unificação', ,Discípulos de Rajneesh' und Seicho-no-iê', die seit Ende der 60er Jahre über nordamerikanische Umwege nach Brasilien gelangten. Ferner sind auch der Islam, das Judentum und der orthodoxe Katholizismus, die mit verschiedenen Einwanderergruppen gekommen sind, in Brasilien präsent.

Dieser knappe Überblick über die gegenwärtig existierenden religiösen Strömungen und Organisationen zeigt eine beträchtliche religiöse Pluralität, so daß der Katholizismus in heutigen Brasilien nicht mehr mit dem Anspruch einer exklusiven Vermittlungsinstanz zwischen den Menschen und dem Göttlichen auftreten kann.

\section{Einige Daten zu Geschichte und Gegenwart der brasilianischen Medienlandschaft}

Die Anfänge des brasilianischen Massenmediensystems gehen zurück auf den Zeitraum zwischen 1920 und 1940, obwohl sich die brasilianische Kulturindustrie erst im Verlauf der 60er Jahre allgemein zu konsolidieren vermochte. Im folgenden will ich einige Zahlen zum Bereich der Printmedien, zum Radio-Markt und zur Entwicklung des Fernsehens vorstellen.

Der Markt der Printmedien (Bücher, Zeitungen und Zeitschriften) entwickelte sich in Brasilien seit den 1930er Jahren. Der Buchsektor wies zwischen 1936 und 1944 eine Steigerungsrate von 46,6\% auf. Zwischen 1944 und 1948 betrug die Wachstumsrate 31\% und hielt sich auf diesem Niveau auch zwischen den Jahren 1948 und 1956. Die Anzahl der 
Verlagshäuser verdoppelte sich zwischen 1936 und 1948, ging dann aber von 280 Verlagen im Jahr 1948 auf nur noch 144 Verlagshäuser im Jahr 1953 zurück. Mit dem Aufbau einer eigenen nationalen Papierindustrie und vor allem mit der 1973 eingeführten Steuerbefreiung für die Produktion und den Verkauf von Büchern erlebte der Buchmarkt dann jedoch trotz einer unverändert hohen Analphabetenquote wieder einen heftigen Aufschwung. So stieg die Buchproduktion von 43 Mio. Exemplaren im Jahr 1966 über 112 Mio. im Jahr 1976 auf 245 Mio. im Jahr 1980; und auch im Zeitschriftenbereich wuchs die Produktion von 104 Mio. Exemplaren im Jahr 1960 über 193 Mio. im Jahr 1970 auf 500 Mio. im Jahr 19854.

In Brasilien bilden jedoch nicht die Printmedien, sondern das Radio das größte und populärste Massenkommunikationsmedium, das heute ca. $90 \%$ aller privaten Haushalte erreicht. Nach seiner Einführung im Jahr 1922 wurden im Verlauf der 20er Jahre 19 Radiosender in verschiedenen Städten des Landes eingerichtet, die jedoch nur die jeweils eigenen Stadtgebiete zu versorgen vermochten. Das vor allem an Gelehrsamkeit, anspruchsvoller Literatur und klassischer Musik orientierte Programm der frühen Radiostationen bestand in der Anfangszeit oft nur aus wenigen täglichen Sendestunden. In dem Maße jedoch, wie die staatliche Rundfunkgesetzgebung auch Radiowerbung zuließ (in den 30er Jahren war der Anteil der Radiowerbung auf $10 \%$ beschränkt; 1952 wurde diese Quote dann auf 20\% der Gesamtsendezeit erhöht), entwickelte sich das Radio allmählich zum wichtigsten masøenmedialen Sektor Brasiliens 5 .

Während in Brasilien 1944 lediglich 106 Radiostationen vorhanden waren, stieg diese Zahl auf $300 \mathrm{im}$ Jahr 1950 und erreichte im März 1993 eine über das gesamte Territorium verteilte Gesamtzahl von 2.784 sendefähigen Radiostationen.

Das Fernsehen wurde in Brasilien in den 50er Jahren eingeführt, war bis 1959 jedoch lediglich eine elitäre technische Errungenschaft für die Oberschichten, da die Empfangsapparate bis zu diesem Zeitpunkt kostspielig importiert werden mußten. Seit Mitte der 60er Jahre wurde dann unter der Militärregierung und ihrer Ideologie der ,Nationalen Sicherheit' der Telekommunikationssektor in Brasilien zielstrebig und mit großem finanziellen Aufwand ausgebaut ${ }^{6}$. In diesem Zusammen-

4 Vgl. dazu R. Ortiz, A moderna tradição brasileira, São Paulo 1988.

5 Vgl. ebd.

6 In diesem Zusammenhang stellt R. Ortiz fest. „1965 wurde die ,Empresa Brasileira de Telecomunicações' gegründet, die die Modernisierung und den Aufbau des Telekommunikationssektors förderte. Im selben Jahr trat Brasilien dem internationalen Satellitensystem (INTELSAT) bei, bevor 1967 ein eigenes Kommunikationsministerium gegründet wurde. Man begann mit dem Aufbau eines landesweiten Mikrowellensystems, das 1968 eröffnet und im Hinblick auf die Amazonasregion im Jahr 1970 vollendet wurde. Damit 
hang läßt sich festhalten, "daß der Staat die technologische Infrastruktur des Telekommunikationssystems bereitstellte und in diesem Sinne die Startbedingungen schuf, die sich dann die privaten Fernsehunternehmen erfolgreich zunutze machen konnten ${ }^{47}$.

Während noch 1970 lediglich 56\% der brasilianischen Haushalte mit Fernsehempfangsgeräten ausgestattet waren, stieg diese Zahl bis zum Jahr 1982 auf $73 \%$. Heute umfaßt Brasilien mit 226 Fernsehstationen ca. 30\% der gesamten lateinamerikanischen Fernsehsender, wobei sich die brasilianische Kulturindustrie immer stärker um das Medium 'Fernsehen' zentriert. Im Brasilien der Gegenwart ist es heute ebenso wichtig, „einen Fernsehapparat im Hause zu haben wie das tägliche Brot auf dem Tisch"8.

Nach dem geltenden brasilianischen Recht liegt die Kontrolle des Telekommunikationsbereichs ausschließlich bei bundesstaatlichen Behörden. Die staatlich verliehenen Sendelizenzen sind im Radiosektor in 10jährigen, im Fernsehbereich in 15jährigen Abständen zu erneuern. Auf dieser gesetzlichen Grundlage verfügt die Zentralregierung faktisch über einen großen politischen Einfluß im Massenmedienbereich, wobei staatliche Stellen nicht selten auch auf der Grundlage persönlicher Interessenlagen Konzentrations- und Monopolisierungsprozesse fördern. $\mathrm{Zu}$ denken wäre hier etwa an die ,Editora Abril', die das größte Verlagsunternehmen Lateinamerikas bildet, und an die ,TV Globo', den viertgrößten Fernsehsender der Welt.

\section{Die römisch-katholische Kirche und die Massenmedien}

Grundsätzlich läßt sich sagen, daß die katholische Kirche den Prozeß der Einführung der Massenmedien in Brasilien von Anfang an begleitet hat. Dies zeigt sich z. B. daran, daß schon 1951 die ,Central Católica de Cinema' gegründet wurde, der dann 1958 die "Rede Nacional de Emissoras Católicas' und 1961 die ,União Católica de Imprensa' nachfolgten. 1962 hat dann die brasilianische Bischofskonferenz (CNBB) ein nationales Sekretariat für Publizistik (Secretariado Nacional de Opinião Pública) gegründet, um die Arbeit der drei bereits existierenden katholischen Medienorganisationen zu koordinieren. Daneben entstand schon früh in vielen Diözesen das Interesse an Aufbau und Ausbildung einer spezialisierten Gruppe kirchlicher Kommunikatoren, so daß sich feststellen läßt, daß die katholische Kirche an dem Siegeszug der

war eine das gesamte Territorium umfassende Kommunikationsinfrastruktur errichtet. Dadurch waren zugleich die technologischen Schwierigkeiten, mit denen sich das Fernsehen in den 50er Jahren konfrontiert sah, gelöst" (ebd., 117f).

7 Ders., Cultura brasileira e identidade nacional, São Paulo 1986, 88.

- M. das Graças e Silva, A Igreja e a evangelização pela TV, São Paulo 1991, 37. 
brasilianischen Kulturindustrie in den 60er und 70er Jahren teilnahm und wesentliche Beiträge zur Entwicklung der brasilianischen Kultur auf diesem Sektor beisteuerte.

Im Hinblick auf den Buchsektor läßt sich der seit den 70er Jahren einsetzende Boom auch im Hinblick auf Produktion und Verbreitung des katholischen Buches nachweisen. Dennoch verliefen Aufbau und Entwicklung des katholischen Verlagswesens eher langsam und unabhängig vom säkularen Buchmarkt, da sich die Produktion des katholischen Schrifttums vor allem an den Bildungs- und Erziehungsaufgaben der verschiedenen Orden orientierte, die bereits seit Ende des 19. Jahrhunderts zu diesen Zwecken eigene Zeitschriften und Wochenblätter produzierten 9 . Trotz dieser frühen Projekte sind die ersten nennenswerten kirchlichen Verlagsaktivitäten, die über den kirchlichen Bereich hinaus auch für den brasilianischen Buchmarkt insgesamt eine Rolle spielten, erst seit den 50er Jahren festzustellen.

1963 wurde von den Jesuiten die "Editora Loyola' gegründet, um zunächst vor allem didaktisches Material für die Ehevorbereitungspastoral und für die Jugendbildung herzustellen. In der letzten Zeit engagiert sich der Verlag dagegen vor allem für kirchennahe Laienbewegungen wie die Cursilhos des Glaubens und die Charismatische Erneuerung. 1964 entstand die "Editora Salesiana Dom Bosco', die vor allem katechetische Bücher produziert; und ein Jahr später folgte die „Editora Frère Théophane Durand' (FTD) der Maristenbrüder, die sich mit didaktischen Schriften befaßt.

Die gegenwärtig bedeutsamsten katholischen Verlagshäuser bilden die 1915 gegründete ,Editora Paulinas' und die 1901 entstandene "Editora Vozes'. Der Vozes-Verlag hat seinen Stammsitz in Petrópolis im Bundesstaat Rio de Janeiro und besitzt zur Zeit 20 Filialen im gesamten brasilianischen Bundesgebiet. Der Paulinas-Verlag hat seinen Hauptsitz in São Paulo und verfügt ebenfalls über 20 Filialen innerhalb und außerhalb des Landes. Der Anteil religiöser Buchtitel beim VozesVerlag, der mit der ,Revista Eclesiástica Brasileira' und der „Revista de

9 Die Salesianer gründeten bereits 1883 ein erstes Verlagshaus in Niterói bei Rio de Janeiro; die Claretiner begannen 1898 mit der Veröffentlichung ihrer Zeitschrift ,Ave Maria'; die Redemptoristen begannen 1900 in Aparecida do Norte (im Bundesstaat São Paulo) mit der Produktion der Wochenzeitung , 0 Santuário'; und schließlich begannen die Franziskaner in Salvador (Bahia) zu Beginn des Jahrhunderts mit der Herausgabe der Zeitung,$O$ mensageiro da Fé' und gründeten 1901 in Petrópolis (bei Rio de Janeiro) eine Druckerei, aus der das heutige Verlagshaus, 'Vozes' hervorgegangen ist; die Kongregation der Pauliner begann 1929 und 1931 mit dem Vertrieb "guter Lektüre” (boa leitura) und publizierte vor allem die Wochenblätter ,La Sequilla' und „Familia Cristă"“ (vgl. P. Montero/R. Della Cava, E o verbo se faz imagem: Igreja Católica e os meios de Comunicação no Brasil: 1962-1989, Petrópolis 1991). 
Cultura Vozes' zwei bedeutsame katholische Zeitschriften herausbringt, liegt gegenwärtig bei ca. $50 \%$ der Gesamtproduktion. Die Veröffentlichungen des Paulinas-Verlages sind dagegen nahezu vollständig religiöser Natur, wobei es schwerpunktmäßig um die Verbreitung der Bibel und um katechetisches Material geht. In gewisser Hinsicht haben sich beide Verlagshäuser auf einen spezifischen Sektor des religiösen Marktes spezialisiert: „Ein Großteil der bei Vozes erscheinenden Titel wendet sich an intellektuelle Eliten, die am gesellschaftlichen Fortschritt und an neuen sozialen Fragen interessiert sind; die ,Editora Loyola' hat ihr Zielpublikum in urbanen Mittelklassen, die sich um an Rom orientierte katholische Laienbewegungen gruppieren; der PaulinasVerlag richtet sich dagegen vor allem an Pastoralagenten und kirchliche Mitarbeiter, die organisatorisch mit den ortskirchlichen Institutionen eng verbunden sind." 10 Der Vozes-Verlag erreichte 1987 den dritten Platz in der Liste der 100 größten brasilianischen Verlagshäuser; noch vor dem Paulinas-Verlag, der Platz 4 einnahm. Damit wird deutlich, daß diese beiden großen Verlage nicht nur einen Großteil des katholischen Buchmarktes beherrschen, sondern sich auch erfolgreich im nicht-religiösen Marktsegment zu behaupten vermögen.

Auch wenn die Produktion religiöser Titel im Steigen begriffen ist, ist sein Marktanteil an der Gesamtproduktion des brasilianischen Verlagswesens eher gering. Dieser Anteil, der von 6,5\% im Jahr 1973 auf 12,5\% im Jahr 1982 anstieg, markiert aber dennoch eine beträchtliche Präsenz des katholischen Verlagswesens in und für die brasilianische Gegenwartskultur.

Die schon im 19. Jahrhundert aufkommende katholische Zeitung bildete lange Zeit eines der wichtigsten Instrumente zur Stärkung und Festigung des kirchlichen Lebens. Mit Ausnahme des ,O São Paulo', der eine wöchentliche Auflage von 15.000 Exemplaren hält, ist die Auflagenhöhe der kirchlichen Zeitungen seit den 40er Jahren jedoch kontinuierlich rückläufig und bewegt sich in einer Größenordnung von 1.000 bis 5.000 Exemplaren pro Titel. Gegenwärtig gibt es 145 katholische Zeitungen mit einer Gesamtauflage unter einer Million Exemplaren. Von diesen 145 Titeln gehören $64 \%$ zu religiösen Ordensgemeinschaften und 36\% zu einzelnen Diözesen bzw. Pfarrgemeinden. Die Zahl katholischen Laien-Zeitungen fällt kaum ins Gewicht.

Ein weiteres wichtiges katholisches Presseprodukt bilden die oft nur aus wenigen Seiten bestehenden sog. ,boletins', die vor allem als diözesane Mitteilungsblätter und als Presseorgane von sozialen Bewegungen und pastoralen Schwerpunktinitiativen fungieren. Sie entstanden seit den frühen 60er Jahren und umfassen gegenwärtig ca. 3.000 Titel mit einer etwa doppelt so hohen Auflage wie die katholischen Zeitungen.

10 Vgl. ebd., 193. 
Im Hinblick auf die elektronischen Medien läßt sich feststellen, daß die katholische Kirche stärker im Radio als im Fernsehbereich involviert ist. Lediglich der Franziskanerorden verfügt über zwei Erfahrungswerte als Eigentümer eines Fernsehsenders, denn zwischen 1969 und 1979 besaßen die Franziskaner die Mehrheitsanteile an der ,TV Difusora' (Porto Alegre); und 1987 erhielten sie die Lizenz für einen regionalen TV-Sender, die 'TV Celinauta' in Pato Branco (Parana), die das Programm eines in Rio de Janeiro ansässigen Privatsenders ausstrahlt und lediglich einige Stunden der wöchentlichen Sendezeit für religiöse Erziehungsprogramme, kirchliche Nachrichten und Gottesdienstübertragungen freihält.

Ein Engagement der Kirche als Eigentümerin von Fernsehsendern ist im Brasilien der Gegenwart also nahezu inexistent. Das bedeutet jedoch nicht, daß sie in diesem Medium nicht präsent ist, denn die Kirche erhält in 32 privaten Fernsehkanälen kostenlose Sendezeit zur Übertragung von Sonntagsgottesdiensten. Darüber hinaus hat an Sonntagen fast das gesamte Land die Möglichkeit, das von Padre Eduardo Dougherty produzierte halbstündige charismatische Programm ,Anunciamos Jesus' (Wir verkündigen Jesus) zu empfangen, wobei die jeweiligen Sendeplätze in diesem Fall jedoch angekauft werden müssen.

Im Hinblick auf das Radio hat die Kirche dagegen bereits eine fast 40jährige Erfahrung aufzuweisen. Das kirchliche Radioengagement, das auf die 40er Jahre zurückgeht, erreichte seinen Höhepunkt zwischen den Jahren 1954 und 1964, in denen - parallel zum boomenden Radiomarkt - die meisten katholischen Rundfunksender gegründet wurden. Heute sind 122 katholische Radiostationen mit überwiegend lokaler Reichweite in Betrieb. Die beiden größten Sender sind ,Radio América' und ,Radio Aparecida'. "Radio América' wurde 1967 von den Pauliner-Padres in São Paulo gekauft und sendet heute ca. acht Stunden pro Woche ein religiöses Programm, was etwa 6\% des vor allem aus populärer Musik bestehenden Gesamtprogramms ausmacht. Seit 1979 gehört "Radio América' zu den drei Radiostationen mit der größten Hörerschaft in São Paulo. ,Radio Aparecida' gehört dem Redemptoristen-Orden und wurde 1951 im Hinblick auf die Bedürfnisse des großen Wallfahrtsortes Aparecida gegründet, an dem die brasilianische Nationalheilige Nossa Senhora Aparecida verehrt wird. "Radio Aparecida' verfügt über einen Kurzwellensender mit nationaler Reichweite und bietet im Gesamtprogramm neben Musik (42,5\%) und Werbung (25\%) ca. $18,5 \%$ religiöse Programme, 6,5\% Regierungsmitteilungen und 5,1\% Nachrichten ${ }^{11}$.

Da sich das Programmangebot der meisten katholischen Radiosender nur wenig von anderen kommerziellen Radiostationen unter-

11 Vgl. dazu P. Montero/R. Della Cava, A Igreja Católica e os meios de Comunicação de massa, in: Religião e Sociedade 13 (1986)3, 62-77, 72. 
scheidet, bemüht sich die brasilianische Bischofskonferenz in den letzten Jahren verstärkt um den Aufbau eines nationalen Netzwerkes katholischer Rundfunksender, um der kirchlichen Radioarbeit dadurch ein stärkeres eigenes Profil zu sichern.

Im audiovisuellen Bereich hat die Kirche ihre ersten Initiativen durch einige Ordensgemeinschaften in den 60er Jahren begonnen und dann seit den 80er Jahren mit steigender Tendenz auch auf den Videobereich ausgedehnt. Im Zentrum dieser Bemühungen steht eine sowohl an die Laien wie auch an den eigenen Klerus gerichtete Bildungsarbeit, die eine wichtige Rolle in der Aus- und Fortbildung pastoraler Mitarbeiter und in der Bewußtseinsbildungsarbeit der Kirche spielt.

\section{Die historischen protestantischen Kirchen Brasiliens und die Massenmedien}

Traditionell unterhalten die protestantischen Kirchen bekanntlich eine enge Verbindung zu den Schriftmedien, was sich auch in Brasilien nachweisen läßt. Die evangelische Kirche lutheranischen Bekenntnisses (Igreja Evangélica de Confissão Luterana no Brasil [IECLB]) ist 1824 mit der Ankunft deutscher Immigranten gegründet worden. Die erste lutheranische Kirchenzeitung wurde 1868 in São Leopoldo als deutschsprachiges Blatt mit dem Titel Sonntagsblatt - für die evangelischen Gemeinden in Brasilien' gegründet. Die Auflage dieser Wochenzeitung, die im Laufe der Jahre mehrere Male den Namen wechselte, stieg von 2.450 Exemplaren im Jahr 1924 über 8.100 im Jahr 1957 auf eine Auflagenzahl von $10.000 \mathrm{im}$ Jahr 1970. 1971 fusionierte diese Zeitung mit anderen evangelischen Zeitungen zum 'Jornal Evangélico', auf das später noch zurückzukommen sein wird.

Ein weiteres wichtiges Blatt der IECLB war der 1908 in Blumenau (Santa Catarina) gegründete ,Christenbote', der mit mehrmaligen Namenswechseln anfänglich eine Auflagenhöhe von ca. 3.000 Exemplaren erreichte und ebenfalls 1971 im Jornal Evangélico' aufging. Zu diesem Zeitpunkt hatte der "Christenbote' eine Auflage von 8.500 Exemplaren. Ferner erschien zwischen 1935 und 1939 der "Heimatbote', der 1951 als ,Neuer Heimatbote' wieder zum Leben erweckte wurde und im Jahr 1965 einen Abonnentenstamm von 2.350 Beziehern erreichte, bevor er 1971 bei einem Stand von 2.100 Bezieher ebenfalls sein Erscheinen einstellte. Die vierte wichtige Zeitung der IECLB ist das 1936 von der evangelischen Gemeinde in São Paulo und Mariana gegründete „Kreuz im Süden".

Während bis zum II. Weltkrieg fast alle Zeitschriften der IECLB ausschließlich die deutsche Sprache benutzten, erscheint diese Presse bis heute in der Regel in zweisprachiger Form. 
Am 15.11.1971 wurden die regionalen IECLB-Zeitungen eingestellt zugunsten einer neuen nationalen evangelischen Kirchenzeitung, dem Jornal Evangélico', um die nationale Einheit der Kirche zu stärken. Das in der 'Editora Synodal' in São Leopoldo produzierte Blatt erscheint im 14tägigem Turnus und setzt mit einer Auflage von 10.000 Exemplaren die 105jährige Tradition der lutherischen Presse in Brasilien fort ${ }^{12}$. Da das Jornal Evangélico' jedoch nicht genügend Aufmerksamkeit auf regionale Ereignisse richten konnte, verlor es im Laufe der Zeit an Abonnenten und förderte den Wiederaufbau regionaler Kirchenzeitungen, so daß im Verlauf der 80er Jahre in Blumenau die Zeitung, $O$ Caminho' und in Vitória (Espirito Santo) die Zeitung ,O Semeador' neu gegründet wurden.

Die IECLB verfügt über drei Verlagshäuser, wobei neben der ,Sinodal', die als ihr größtes Unternehmen auch auf dem nationalen Markt tätig ist, die ,Luz e Vida' in São Paulo und die ,União Cristä' in Curitiba (Parana) zu nennen sind ${ }^{13}$.

Im elektronischen Bereich besitzt die IECLB drei Radiosender in Novo Hamburgo, in Blumenau und in Florianópolis sowie eine 1975 gegründete Schallplattenfirma im Porto Alegre. Ca. 15\% des täglichen Programmangebots dieser Radiostationen hat religiösen Charakter. Darüber hinaus verbreitet die IECLB über viele private Radiostationen im Landesinnern das aus Meditation, Hymnen und sozialen bzw. kirchlichen Nachrichten bestehende Programm ,Hora Evangélica' (Evangelische Stunde). Ein ähnliches Programm findet sich auch gelegentlich in einigen privaten Fernsehstationen.

Neben der IECLB ist die ,Igreja Evangélica Luterana do Brasil (IELB) zu nennen, die auf die Synode von Missouri'14 zurückgeht und 1902 in Brasilien gegründet worden ist. Sie verfügt mit der ,Editora Concordia' in Porto Alegre über ein wichtiges Verlagshaus und vertreibt auf nationaler Ebene mit dem 1917 gegründeten "Mensageiro Luterano' ihre wichtigste Zeitung mit einer aktuellen Auflage von 11.000 Exemplaren. Die IELB besitzt darüber hinaus in Canoas (Rio Grande do Sul) eine eigene Radiostation und kauft bei ca. 150 privaten Radiostationen Sendeplätze ein, um zwei Programme, die ,Hora Luterana' und ,Cinco minutos com Jesus' (Fünf Minuten mit Jesus) zu verbreiten. Die Präsenz von IELB-Programmen im Fernsehen ist dagegen bis heute nur

12 Im Jahr 1973 verzeichnete das Jornal Evangélico' einen Abonnentenstamm von 23.498, sankt dann bis zum Jahr 1981 jedoch auf die Zahl von 22.000 festen Beziehern.

13 Vgl. I. Starke, A Imprensa escrita da Igreja Evangélica de Confissão Luterana no Brasil, São Leopoldo 1981.

14 Vgl. zu der 1847 von sächsischen Immigranten in Nordamerika gegründeten ,Deutschen Evangelisch-Lutherischen Synode von Missouri, Ohio und anderen Staaten' H.-J. Prien, Die Geschichte des Christentums in Lateinamerika, Göttingen 1978, 767ff. 
sehr schwach. Beide lutheranischen Kirchen geben gemeinsam das jährlich Losungsbuch ,Castelo Forte' heraus, das sich zur Zeit im 26. Jahrgang befindet und eine Auflage von 60.000 Exemplaren erreicht.

Die methodistische Kirche mit Sitz in São Paulo gibt die Monatszeitung ,Expositor Cristão' mit einer Auflage von 10.000 Exemplaren heraus. Auch hier hat jede Region ihre eigene Zeitung. Die methodistische Kirche besitzt weder Radio- noch Fernsehstationen, verfügt aber über angekaufte Sendeplätze in privaten Fernsehsendern.

Die Baptisten vertreiben mit dem 1909 gegründeten Jornal Batista' auf nationaler Ebene eine wöchentliche Zeitung, die gemeinsam mit dem nur in Rio Grande do Sul erscheinenden ,Batista Gaúcho' (seit 1948) eine Auflagenhöhe von 10.000 Exemplaren erreicht. Die Baptisten vertreiben darüber hinaus eine Vielzahl weiterer Zeitschriften wie, 0 Homen Batista', ,Visão Missionário' ,Revista da Juventude', ,O Jovem Casal' und 'Realização'. Seit 1983 verfügt die baptistische Kirche mit dem in Rio de Janeiro angesiedelten Fernsehkanal ,TV Rio' und dem ,Radio Relógio' über eigene elektronische Medien. Daneben erreicht sie auch über angekaufte Sendeplätze in vielen privaten Radios landesweit eine hohe massenmediale Präsenz.

Im Unterschied dazu besitzt die presbyterianische Kirche von Brasilien mit dem , $O$ Brasil Presbiteriano' lediglich eine landesweite Zeitung, die sich gegenwärtig in einer schweren Finanzkrise befindet, von der auch das in São Paulo angesiedelte Verlagshaus ,Casa Editora Presbiteriana' in Mitleidenschaft gezogen ist. Die Presbyterianer, die über keine eigenen Radio- und Fernsehsender verfügen, erwerben ebenfalls Sendeplätze in privaten Radiostationen für ihr meist halbstündiges Programm ,Luz para o caminho' (Licht für den Weg). Die Präsenz der presbyterianischen Kirche im Fernsehsektor ist ebenfalls nur äußerst spärlich.

\section{Die pfingstlerischen Sekten in Brasilien und die Massenmedien}

Im Brasilien der Gegenwart scheinen lediglich die protestantischen Sekten ein größeres Gewicht auf die neuen elektronischen Medien als auf die älteren Printmedien zu legen.

Die größte pfingstkirchliche Denomination Brasiliens bildet die aus Nordamerika stammende und 1911 in Brasilien gegründete ,Assembléia de Deus', die heute (nach eigenen Angaben von 1992) über ca. 12 Mio. Anhänger verfügt. Im Printmedienbereich ist neben der älteren Zeitung ,Boa Semente' heute vor allem der ,Mensageiro da Paz' zu nennen, der mit einer Auflage von über 500.000 Exemplaren das wichtigste Massenmedium dieser Kirche ausmacht. Neben dieser, im eigenen Verlagshaus in Rio de Janeiro, der ,Casa Publicadora', gedruckten Zeitung erscheinen mit geringerer Auflage noch "Nosso Lar', 
Seara' und ,Obreiro'. Die ,Assembleia de Deus' besitzt keine eigenen Radiostationen, strahlt jedoch täglich ca. 2.000 religiöse Programme über private Radiostationen aus und verbreitet in den meisten brasilianischen Bundesstaaten über private Fernsehkanäle ein wöchentliches religiöses Fernsehprogramm.

Die ,Congregação Cristã do Brasil' wurde 1910 in Brasilien gegründet und hat sich ebenfalls im ganzen Land ausgebreitet, obwohl sie bis heute sowohl im Druck- als auch im elektronischen Medienbereich nur ein sehr geringes Engagement entwickelt.

Die 1940 aus den Vereinigten Staaten importierte ,Igreja do Evangelho Quadrangular' entfaltet dagegen eine breite Präsenz im Radiobereich und sendet täglich ca. 400 religiöse Programme.

Neben diesen traditionellen Pfingstkirchen sind es vor allem die einheimischen Pfingstsekten, die sog. neo-pentekostalistischen Denominationen, die heute mit Nachdruck in den Radio- und Fernsehbereich drängen. Hier sind besonders die ,Igreja Universal do Reino de Deus' und die ,Igreja Deus é Amor' zu nennen.

Die ,Igreja Universal do Reino de Deus' (Universale Kirche vom Reiche Gottes) wurde 1977 in Rio de Janeiro gegründet und verfügt heute über mehr als 1.000 Tempel im ganzen Land. Sie ist gegenwärtig in 20 Ländern in Lateinamerika, Afrika, Europa und Nordamerika präsent. Von Anfang an spielte die elektronische Massenpredigt eine besondere Rolle in der ,Igreja Universal do Reino de Deus'. Sie besitzt heute nicht nur 12 eigene Radiostationen, sondern verfügt mit der ,TV Record' in São Paulo auch über einen großen und wichtigen Fernsehsender. Dennoch ist auch hier der Anteil der religiösen Programme an der Gesamtsendezeit eher gering. Die ,Igreja Universal do Reino de Deus' verbreitet ihre Botschaften nicht nur über eigene elektronische Medien, sondern ähnlich wie die anderen Religionen auch über die Anmietung einer Vielzahl von Sendeplätzen in verschiedenen Radio- und Fernsehsendern. In den größten und wichtigsten brasilianischen Bundesstaaten sendet sie täglich das Morgenprogramm, $O$ Despertar da Fè'. Ferner verfügt diese Kirche mit der ,Universal Produçōes' über einen eigenen Verlag, der neben Büchern und Boletins die Zeitung "Folha Universal" und die Monatszeitschrift "Plenitude' mit einer Gesamtauflagen von 200.000 Exemplare vertreibt.

Die ,Igreja Deus é Amor' (Kirche Gott ist Liebe) wurde 1962 in São Paulo gegründet und verfügt gegenwärtig über ca. 600 Gotteshäuser in ganz Brasilien. Es handelt sich ebenfalls um eine expandierende Sekte, die sich zur Zeit in verschiedenen Ländern Lateinamerikas und in den Vereinigten Staaten ausbreitet. Sie besitzt fünf Radiostationen, mit deren Hilfe sie das Programm ,A Voz da Libertação' (Stimme der Befreiung) verbreitet, das in Sāo Paulo produziert wird und in ganz Brasilien und auch in anderen lateinamerikanischen Ländern ausge- 
strahlt wird. Aktivitäten im Fernsehsektor werden von dieser Sekte jedoch bis heute nicht aufgenommen.

Weitere neo-pentekostalistische Sekten entfalten ebenfalls ein beträchtliches Engagement im Bereich der elektronischen Medien. Zu nennen wären etwa die Kirche,$O$ Brasil para Cristo' (Brasilien zu Christus), die täglich 280 religiöse Programme über ca. 250 Radiostationen verbreitet; die ,Igreja Evangelica Pentecostal Cristã', die ebenfalls mit täglichen Radio- und Fernsehprogrammen in Südbrasilien präsent ist; die ,Igreja Internacional da Graça de Deus' (Internationale Kirche von der Gnade Gottes) und die ,Igreja Casa da Bênção' (Kirche Haus des Segens), die ebenfalls im Radiobereich engagiert sind.

\section{Abschließende Bemerkungen}

Im Anschluß an diesen Überblick über die Präsenz der verschiedenen christlichen Religionen in der aktuellen brasilianischen Medienlandschaft lassen sich die wichtigsten Ergebnisse in einigen knappen Aussagen zusammenfassen. festhalten:

Im Hinblick auf die katholische Kirche läßt sich folgendes

a) Unter den katholischen Verlagshäusern haben sich ,Vozes' und ,Paulinas' einen festen Platz auf dem brasilianischen Buchmarkt erobert, weil sie neben einer guten internen Organisationsstruktur ihre Produktpalette auch auf den profanen, speziell den universitären Bereich ausgedehnt haben;

b) im Gegensatz dazu ist das katholische Zeitungswesen nicht hinreichend in der Lage gewesen, die Entwicklungen und Veränderungen hin zu einem modernen Journalismus mitzumachen, wodurch sich sowohl im Hinblick auf die professionellen Standards als auch hinsichtlich der technologischen Entwicklung beträchtliche Defizite nachweisen lassen;

c) ähnlich ist auch die Zahl der katholischen Radiosender aufgrund technischer Rückständigkeit und mangelnder finanzieller Ausstattung, die auch mit einer geringen Bereitschaft zu bischöflicher Finanzunterstützung in diesem Bereich zusammenhängt, seit langem rückläufig;

d) die beiden großen und erfolgreichen katholischen Radiosender "Radio Aparecida' und ,Radio América" folgen in ihrer Entwicklung in gewisser Weise dem Modell der pfingstlerischen Radiostationen, da sie sich mit ihren Programmschwerpunkten ebenfalls vor allem an den Bedürfnissen der unteren Volksklassen orientieren; 
e) die Präsenz der Kirche im Fernsehsektor ist sehr bescheiden und beschränkt sich nahezu vollständig auf die Übertragung von Sonntagsgottesdiensten;

f) obwohl die Bischofskonferenz ausdrücklich die Bedeutung des Gebrauchs und des Besitzes von elektronischen Massenmedien anerkennt, herrschen bis heute Zweifel und Unsicherheit hinsichtlich der konkreten Formen eines kirchlichen Medienengagements vor.

Im Hinblick auf die historischen protestantischen Kirchen sind folgende Beobachtungen festzuhalten:

a) Aufgrund der hohen Bedeutung, die die evangelische Glaubenstradition dem Medium der Schrift zuspricht, kommt den evangelischen Kirchen faktisch eine besondere Rolle für Entstehung und Entwicklung der Printmedien in Brasilien zu. Eine genauere historische Analyse würde diese Zusammenhänge sicherlich näher aufweisen können;

b) die Präsenz dieser Kirchen im elektronischen Medienbereich ist dagegen sehr jungen Datums und bis heute relativ bedeutungslos geblieben.

Die pfingstlerischen bzw. neo-pfingstlerischen Sekten kennzeichnen sich dagegen durch eine klare Option für die elektronischen Medien; und zwar in mehrfacher Hinsicht: Zunächst und vor allem bilden sie natürlich das zentrale Medium für ihre Missionierungs- und Erweckungsbemühungen; ferner bilden die elektronischen Medien aber auch eine wichtige ökonomische Einnahmequelle, da die religiösen Programme durchgängig von regelmäßigen Spendenaufrufen durch die Prediger und Pastoren durchsetzt sind, um die hohen Produktions- und Vertriebskosten des umfangreichen religiösen Programmangebots finanzieren zu können; und schließlich fungiert ein erfolgreiches Engagement in Empfang, Verbreitung und Verwendung elektronischer Massenmedien bei den überwiegend aus sozial schwachen Bevölkerungsschichten rekrutierten Mitgliedern der pfingstlerischen Sekten auch als ein wichtiges Statusobjekt für das Sozialprestige dieser neuen religiösen Bewegungen, die sich dadurch zugleich als besonders modern und zeitgemäß erleben können.

\section{SUMMARY: Christian religions and mass media in Brazil}

The author gives a brief overview on the correlation between the different Christian religions of Brazil and modern mass media.

He starts off with some information on the history and present situation of the existing religions in Brazil. Afterwards the basic data of the Brazilian media system is pointed out. On this foundation he describes in detail the ways in which the Roman Catholic Church uses mass media and what is about the media presence of Catholicism in the present Brazilian culture. Likewise, the 
different Protestant Churches are portrayed in detail. In this case, the attention is not only on traditional Protestant denominations, which go back to the era of the Reformation, but also on the development of the Pentecostal and Neo-Pentecostal movements of the 20th century, which, in the last decades, can report rapid growing rates in Brazil.

\section{RÉSUMÉ: Les religions chrétiennes et les masse-médias au Brésil}

L'auteur nous livre un court aperçu sur la relation entre les différentes religions chrétiennes et les médias modernes au Brésil.

Tout d'abord, il donne quelques informations sur l'histoire et le présent des religions qui sont actuellement représentées au Brésil. Ensuite, il nous fourni des informations de base en ce qui concerne le système brésilien des médias. $A$ partir de cela, il décrit d'une façon très détaillée le comportement et l'emploi des masse-médias par l'eglise catholique-romaine ainsi que la présence médiatique du catholicisme dans la culture contemporaine brésilienne. Les différentes églises protestantes sont présentées d'une façon tout aussi détaillée. Il ne fait pas seulement attention aux dénominations protestantes traditionnelles qui ramènent à l'époque de la Réformation, mais aussi aux développements des mouvements pentecôtistes et néopentecôtistes du 20 ème siècle qui présentent des taux d'accroisements rapides dans les dernières décennies au Brésil.

\section{Brasil}

RESUMEN: Religiones cristianas y medios de comunicación en

El autor nos proporciona una concisa visión de la relación de las diferentes religiones cristianas de Brasil con los medios de comunicación de masas modernos.

En primer lugar nos informa de la historia y de la situación actual de aquellas religiones existentes hoy en Brasil. Después nos da a conocer los datos básicos del sistema de comunicación de masas brasileño. Basándose en lo anteriormente mencionado nos hace una detallada exposición del trato y uso que hace la iglesia católica-romana de los medios de masas y la presencia en ellos del catolicismo en el Brasil de hoy. En forma igualmente detallada nos presenta los diferentes iglesias protestantes. El punto de atención no se centra sólo en la tradicional dominación protestante que se remonta al tiempo de la Reforma, sino también en el desarrollo de los movimientos pentecostales y neopentecostales del siglo $\chi \chi$ que en las últimas décadas han tenido un crecimiento muy rápido en Brasil. 